\title{
Manejo integral de desechos domiciliarios para el desarrollo territorial, distrito de Shanao 2019
}

Mg. Bao Ratzemberg, Carol Beatriz

(ORCID: 0000-0003-4205-7446) cbaor@ucvvirtual.edu.pe

Dr. Sánchez Dávila, Keller (ORCID: 0000-0003-3911-3806) ssanchezda2081@ucvvirtual.edu.pe

Dr. Delgado Bardales, José Manuel

(ORCID: 0000-0001-6574-2759) jmdelgadob@ucvvirtual.edu.pe Scopus autor ID: 24070333700 Código Renacyt: P0050554

Dra. Contreras, Rosa Mabel (ORCID: 0000-0002-0196-1351) rcontrerasj@ucv.edu.pe

\section{RESUMEN}

\section{Dra. Palomino Alvarado, Gabriela del Pilar}

(ORCID: 0000-0003-0851-4375) dpalominoal@ucvvirtual.edu.pe Universidad César Vallejo Tarapoto - Perú

Objetivo del estudio fue determinar la relación del manejo de desechos domiciliarios y desarrollo local, municipalidad distrital de Shanao, 2019. El estudio se fundamenta en las teorías y normativa de los residuos domiciliarios y por otro lado el desarrollo local sustentado por Cepal. Estudio de tipo no experimental, diseño descriptivo correlacional. Población y muestra del estudio conformada por 131 ciudadanos del distrito de Shanao. La técnica empleada fue la encuesta y el cuestionario como instrumento. Concluyó que el nivel desechos domiciliarios es "Regular" con 32\% y el nivel de desarrollo local "Bueno" con 31\%; asimismo existe una relación alta positiva entre el manejo de desechos domiciliarios y desarrollo local en la municipalidad distrital de Shanao, sustentado en un coeficiente de Pearson de 0.905 , y un coeficiente de determinación de (0.820), explicando que el $82 \%$ del desarrollo local es influenciado por el manejo de desechos domiciliarios de la municipalidad distrital de Shanao.

Palabras claves: Vivienda, desarrollo local, gobierno local. 


\title{
Comprehensive management of household waste for territorial development, Shanao district 2019
}

\begin{abstract}
Objective of the study was to determine the relationship of household waste management and local development, Shanao district municipality, 2019. The study is based on the theories and regulations of household waste and, on the other hand, local development supported by Cepal. Non-experimental study, descriptive correlational design. Population and sample of the study made up of 131 citizens of the Shanao district. The technique used was the survey and the questionnaire as an instrument. He concluded that the level of household waste is "Regular" with $32 \%$ and the level of local development "Good" with $31 \%$; There is also a high positive relationship between household waste management and local development in the Shanao district municipality, supported by a Pearson coefficient of 0.905 , and a determination coefficient of (0.820), explaining that $82 \%$ of local development is influenced by the household waste management of Shanao District Municipality.
\end{abstract}

Keywords: Housing, local development, local government.

Artículo recibido: 19 enero 2020 Aceptado para publicación: 22 febrero 2020 Correspondencia: cbaor@ucvvirtual.edu.pe Conflictos de Interés: Ninguna que declarar 


\section{INTRODUCCIÓN}

La selva peruana cuenta con la mayor cantidad de ríos que atraviesan las regiones, utilizadas como fuente de abastecimiento para consumo humano, también es utilizado como vía para eliminar elementos de desechos, desde basura a excremento, desde ahí podemos observar la velocidad de contaminación ambiental a través de los ríos y la necesidad de frenar la contaminación, ensayando diferentes propuestas que muchas veces no dan resultados esperados (Escobar, 2003, p. 45). Es así, que se iniciaron a realizar manejo de aguas residuales en grupos poblacionales específicos, mediante el uso de biofiltros, como medios experimentales iniciado en Alemania en los años noventa. Luego está experiencia fue trasladado a diferentes latitudes, como es el caso de una instalación de planta piloto en Masaya, Nicaragua; donde se beneficiaron alrededor de mil ciudadanos (Aguilar, 2014, p. 67).

De la misma manera, países como El Salvador, con apoyo de entes externos (COSUDE) implementaron un sistema de tratamiento ubicado en San José Las Flores, Chalatenango, en donde logro insertar educación ambiental, y la inserción de la ciudanía en el desarrollo del proyecto, garantizando la conclusión y mantenimiento de la intervención (Aguilar, 2014, p. 71). Con los buenos resultados del uso de los biofiltros, los proyectos se desarrollaron en Europa y el mismo Estados Unidos, se empezó a protocolizar y normativizar su uso, llegando a realizar la denominada conferencia sobre biofiltros, siendo Francia el anfitrión de dicho evento junto con la International Water Association (IWA), propiciando un espacio de intercambio de información y experiencias (Buitron, 2014, p. 34).

En lo que respecta la región San Martín, específicamente como Distrito de Shanao, existen plantas de humedales residuales, que tienen propósito en minimizar los efectos contaminantes en el río Mayo, donde la contaminación es consecuente a otros ríos, siendo necesario tomar medidas correctivas, iniciando por las decisiones de las autoridades, ya que ocasiona pérdida de valor económico, porque queda imposibilitado a ser utilizado, perdiéndose valor agregado. Para ello los gobiernos locales y regionales, deben de propiciar centros de producción, para impulsar la comercialización, cualquier tipo de producto, lo que podrían hacer es a través de los pobladores constituir grupos de trabajo y sean ellos llevar a 
comercializar productos, como sería el caso de los abonos, e incluso generar energía eléctrica. Lo mencionado es para funcional de las autoridades de turno, en sus diferentes niveles de toma de decisiones, en donde pueda vislumbrar su desarrollo de manera integral apoyado en los diferentes instrumentos de gestión y que tenga un efecto positivo en la ciudadanía (Marin, Barreix, \& Machado, 2015).

Respecto a los antecedentes, se presenta los hallazgos del nivel internacional, en la que destacan Cueva, E. y Yasmany, F. (2013), quien concluyó, que el tratamiento aplicado tuvo una reducción de un 75\% (DBO5 37 mg/l, DQO 107 mg/l, aluminio 1,037 mg/l, nitrógeno total 9,872 mg/l, índice de Coliformes 1,8 x108, fósforo total 2,38 mg/l, sólidos totales 321 mg/l). Pero no presentó diferencias significativas en los tratamientos, en donde los humedales se están ubicados en rangos adecuados de acuerdo a la norma.

Bernal, J. (2014), concluyó que la tasa de crecimiento, es similar con la tasa de crecimiento real, alcanzo un error de 0,2\% en Asia y América del Sur, 0.1\% para la OCDE, 0.4 África y con $0,1 \%$ alcanzó Colombia. Cabe precisar que de acuerdo al análisis de Harrod, que la tasa de crecimiento garantizada y observada disminuiría la problemática de inestabilidad.

Vinueza, J. (2014), concluyo que un afluente intervenido se verifico la eficiencia del manejo del tratamiento. En el nivel nacional, destaca el estudio de Arce, L. (2013), concluyó que la iniciativa de urbanizaciones sostenibles genera efectos positivos, usando la aireación extendida y los biorreactores de membranas. Baca, M. (2012), concluyó que las concentraciones de DQO y DBO5 y relacionadas DQO/DBO5 muestran un valor fuera del rango, respecto a aguas biodegradables, en donde se encontraría productos no biodegradables, los mismos que están presentes como DQO.

Espinoza, R. (2015), concluyó que el sistema de tratamiento tiene distribución inadecuada, donde presenta irrigación en los desagües con ausencia de tratamiento. Por otro lado, las lagunas primarias tienen añadidura, donde la distribución de sólidos es deficiente, conllevando a un azolvamiento.

Finalmente, en el ámbito local y regional destaca el estudio de Sánchez, L. (2014), concluyendo que la empresa en especial va generando descargas de aguas residuales industriales y domésticas a la quebrada "Zancudo" causando la contaminación de sus aguas produciendo efectos negativos en el medio ambiente. Por otro lado, el estudio identifico la 
ausencia de manejo de aguas residuales. Asimismo, aguas drenadas sin tratamiento por la planta provocan malos olores, proliferación de insectos, teniendo sus consecuencias en la salud.

Lozano, A. (2019), concluyó que el sistema gestión de desechos domiciliarios obtiene un $14 \%$, la gestión integral, buena con 79\%; en donde se identifica procesos, en la que identifica una valoración de regular.

Mori, P. (2018), concluyendo que la recaudación tributaria y el desarrollo local en el distrito de Barranquita se correlación positivamente en un nivel alto.

Seguidamente se plantea las teorías, desarrollando en un primer momento los desechos domiciliarios; donde Montes (2009) refiere que son productos de índole orgánico e inorgánico, los mismos que son descartados al concluir su vida útil (p. 20). MINAM (2016), define a subproductos y/o productos de consistencia semisólida y/o sólida, que el proveedor tiene que desechar de acuerdo a la normatividad vigente y ejercida a nivel nacional (p. 9). Otro autor, refiere que los desechos sólidos en sus diferentes presentaciones, deben ser eliminados tras haberse utilizado (Pinto, 2009, p. 54). En tanto, los desechos sólidos son materiales que tras haberse utilizado pasan a formar parte de desechos orgánicos e inorgánicos, donde su valor se ve afectado, originándose un elemento de alto riesgo para la salud pública y ambiental. Sin embargo, los residuos sólidos pueden adquirir valor al ser manejado adecuadamente. Los residuos sólidos se caracterizan, por su origen, relacionado en donde se produce los desechos, y por la peligrosidad, definidos como peligrosos o no; por su naturaleza, orgánicos e inorgánicos.

De acuerdo a la Ley General de los Residuos Sólidos (2000) $\mathrm{N}^{\circ}$ 27314, refiere que los residuos de gestión municipal, y mediante la D.S. $\mathrm{N}^{\circ}$ 057-2004-PCM, otorga la responsabilidad y competencias a los gobiernos locales sobre los desechos generados en domicilios, comercios, mercados e instituciones (p. 53). Alfaro (2008), hace mención de la responsabilidad del gobierno local del manejo de los desechos generados en la jurisdicción mediante su área correspondiente del gobierno local (p. 24). De acuerdo a lo mencionado, todo gobierno municipal tiene la responsabilidad de gestionar sus residuos sólidos, los mismos que fueron generados por la comunidad, sectores, instituciones y otros que se encuentren en la circunscripción geográfica. 
Por su parte los residuos de gestión no municipal, lo estipula la misma Ley General de Residuos Sólidos, donde su manejo y tratamiento es realizado con altos niveles de seguridad, para minimizar el peligro de contaminación, realizado un manejo integral, con acciones definidas (p. 46). La Dirección General de Políticas (2009), pone de manifiesto una serie de procesos que se articulan, iniciando con la reutilización, reciclaje, tratamiento y comercialización de desechos sólidos, finalmente, el transporte al destino final de los desechos, lugar donde se degradara. En ese sentido, es preciso identificar los procesos que se desarrollan con los desechos domésticos, iniciando con la selección, manipulación, almacenamiento y disposición final; es preciso aclarar que la manipulación y almacenamiento está ligada con el manejo selectivo.

PROARCA (2003), hace mención que el manejo integral de desechos sólidos, donde incopora en primera instancia la clasificación, el empleo de técnicas y el uso de tecnologias apropiadas para realiar un manejo eficiente para conseguir objetivos y lograr metas concretas (p. 5). De acuerdo a lo mencionado, es necesario hacer incapie en la selección de los desechos, emplesr ecnicas adecuadas de la mano con la tecnologia para hacer eficiente el trabajo del gobierno municipal. Karak (2012), plantea los siguientes pasos y/o procesos iniciandose por el recojo, traslado, valorización, reciclaje y tratamiento, culminando en la disposición final; donde menciona que cada proceso desarrollado eficientemente evitara ocasionar perjuicios al medio ambiente (p. 1561).

De acuerdo al D.S. N 057-2004-PCM (2004), aborda sobre el responsable del manejo de los desechos, el mismo que pueden ser el mismo gobierno municipal, de acuerdo a sus competencias y elementos que tiene para hacer un trabajo eficiente, donde también puede ser un tercero, denominada EPS-RS, donde el gobierno llega a un acuerdo entre ambas partes y son firmantes de un contrato, en donde estipula proporcionar la mayor cobertura del servicio, siendo este servicio con características estandarizadas (p. 8). Semarnat (2003), hace referencia que es necesario que los diferentes procesos puedan hacer sinergia en sus diferentes procedimientos, hasta el último que es el confinamiento final; todo ello puede configurar consecuencias positivas ambientales e incluso en la parte económica en las personas involucradas en procesos desarrollados (p. 14-15). 
Al realizar los procesos de los residuos sólidos, se encuentran muchas dificultades debido a la cantidad y a la tipología; a ello se adiciona otros factores externos que dificultan el manejo, con solo mencionar la migración a las zonas urbanas, efectos de la tecnología, consumo de energía, entre otros; y para ser eficiente debe generarse una guía de acuerdo al contexto real del entorno territorial. Sin embargo, el país tiene un accionar lento, con accionar diverso a lo reglamentado, pues es claro ejemplo que pone en riesgo la salud ambiental y de sus habitantes. Aunque, un sector mínimo de la población viene aprovechando el manejo de los residuos domiciliarios, todo ello relacionado con los niveles educativos y socioculturales (PNUD, 2011).

Seguidamente se desarrolla conceptualmente el desarrollo local, que es visto de diferente óptica, de acuerdo a sus propios paradigmas, en donde muchas personas lo consideran como trabajo, salud; todo ello de acuerdo a su concepción (Márquez, 2011). Otra definición conceptual se relaciona con las actividades económicas y sociales enmarcado en un contexto geográfico (Marquez \& Rovira, 2002). En lo que respecta al espacio nacional, para considerar un desarrollo local, debe contener seis ejes estratégicos para realizar una mirada multidimensional, en donde la participación de los gobiernos locales es fundamental, debiendo considerarse a infraestructura y acceso a servicios educativos y sanitarios; en donde los demás ejes deben complementarse para un buen desarrollo (CEPLAN, 2011).

El desarrollo local, es analizado por infraestructura, donde Cepal (2004), menciona que acceder a infraestructura adecuadas, en donde se presta servicios públicos, permitiendo ampliar su cobertura de atención y acceso para los ciudadanos. Caso contrario, contar con infraestructura inadecuada conlleva a dificultades en poder desarrollar políticas, que repercute en la parte socioeconómica. En tal sentido, un país con infraestructura adecuada va más allá de la cobertura de servicios, si no generar una mayor oportunidad y se convierte en una ventaja competitiva (p. 7). Por otro lado, el desarrollo local, se relaciona con la salud, considerando el estado completo de bienestar físico y social, por tanto, no solo la presencia de patologías; donde las necesidades de la salud considerada como un bien público en busca del logro de maximización de bienestar en una determinan circunscripción. Por su parte, la educación, se vincula en el desarrollo de capacidades cognitivas y físicas en beneficio de la 
sociedad. Todo ello, contar con mayor acceso y crecimiento académico en sus diferentes niveles de educación (Cepal - Ilpes, 2003).

Para alcanzar el ansiado desarrollo, tiene que gestarse una articulación gubernamental, con socios estratégicos, involucrando a los sectores públicos, y de la generación de la necesidad proveniente de los ciudadanos. Surgiendo la necesidad de la intervención del estado, para ello la normatividad legal debe ser ejercido de manera equitativa y poder reflejarse en los ciudadanos (USAID, 2011). Asimismo, USAID (2011) considera que para dar sostenibilidad al desarrollo es manejar los tributos, creando, modificando o derogando; para un buen ejercicio de los tomadores de decisiones que beneficien directamente a sus habitantes. Para llegar a la concretización de un verdadero desarrollo local es la generación de inversión pública, siendo este primordial para mejorar la oferta de servicios adecuados, con mayor acceso y de mejor calidad (MEF, 2016). Para ello, es importante el accionar técnico político de los decisores para minimizar las externalidades negativas en la población (IFC \& ACDI, 2012).

Los gobiernos locales priorizan su intervención en sus territorios en los ejes económicos y sociales, para ello es necesario diseñar planes económicos, vinculado con planes del nivel nacional y regional; en tanto, el plan concertado debe garantizar, e involucrar a los ciudadanos para involucrar en el desarrollo local. En tanto, la parte del desarrollo económico debe enfatizar en la valorización del sector empresarial en sus diferentes formas, para el desarrollo social, se relaciona con las capacidades de sus pobladores y la equidad en accesibilidad a servicios públicos (IFC \& ACDI, 2012). De lo mencionado se planteó como problema ¿Cuál es la relación del manejo de desechos domiciliarios con el desarrollo local en la Municipalidad Distrital de Shanao, 2019? Como problemas específicos ¿Cuál es el nivel de manejo de desechos domiciliarios en la municipalidad distrital de Shanao, 2019? ¿Cuál es el nivel de desarrollo local en la municipalidad distrital de Shanao, 2019?

Respecto a la justificación; por conveniencia, porque minimizó la contaminación ambiental, mediante la aplicación de manejo eficiente de los residuos domiciliarios, replicando a distritos aledaños para insertar en planes de gobierno municipal direccionado al manejo de residuos domiciliarios y contaminación ambiental. Relevancia social, permitió generar conciencia en la ciudadanía y con ello evitar enfermedades provenientes de la contaminación 
de los residuos domiciliarios que afectan en mayor proporción a los niños. Valor teórico, permitió conocer los diferentes significados y su relevancia que tiene la contaminación ambiental y el tratamiento de residuos sólidos, generando conocimiento mediante los hallazgos propios del estudio. Implicancia práctica, permitió realizar un aporte al gobierno local para propiciar medidas correctivas de mediano y largo plazo, el mismo que beneficiara a la comunidad. Utilidad Metodológica, contribuyó a generar una experiencia que pueda ser replicada en otros distritos de la provincia de Lamas.

El estudio definió al objetivo general, determinar la relación del manejo de desechos domiciliarios y desarrollo local, Municipalidad Distrital de Shanao, 2019. Como objetivos específicos, determinar el nivel de manejo de desechos domiciliarios por la municipalidad distrital de Shanao, 2019, y establecer el nivel de desarrollo local en la municipalidad distrital de Shanao, 2019.

\section{ESTRATEGIAS METODOLÓGICAS O MATERIALES Y MÉTODOS}

La investigación fue no experimental, identificándose por la nula manipulación de variables estudiadas; asimismo, tiene como fin visualizar diferentes fenómenos en su contexto usual (Hernández Sampieri, 2016). Ejecutó un diseño descriptivo correlacional, que mide asociación de variables, es decir la importancia que tiene una sobre la otra (Hernández Sampieri, 2016). La población estuvo conforma por habitantes del distrito de Shanao, en relación al registro del INEI, correspondiente a 2089 habitantes (INEI, 2007). La muestra estuvo constituida por 131 habitantes, tras la aplicación de la fórmula de población finita (INEI, 2007). Se utilizó el aleatorio simple. Como criterios de inclusión se consideró que los ciudadanos del distrito de Shanao, principalmente jóvenes, adultos y adultos mayores. Ciudadanos que conocen temas relacionados a contaminación ambiental y pobladores involucrados en la conservación del medio ambiente. La técnica empleada fue la encuesta, facilitando acopiar datos proporcionados por pobladores del distrito, garantizando con ello fidedignidad de la información. Para la construcción del instrumento de desecho domiciliario, se apoyó en el estudio de Lozano (2019). Para la construcción se consideró tres dimensiones, segregación domiciliaria, tratamiento y comercialización; donde se utilizó cinco ítems por dimensión, los puntajes oscilan entre 1 a 5 puntos, iniciando con calificación pésima, deficiente, regular, bueno y el máximo valor (5) excelente. En cuento a la 
construcción del instrumento de desarrollo local, se basó en el estudio de Mori (2018). El instrumento tuvo como dimensiones, infraestructura, salud y educación; donde se utilizó cinco ítems por dimensión, los puntajes oscilan entre 1 a 5 puntos, iniciando con calificación pésima, deficiente, regular, bueno y el máximo valor (5) excelente. Es importante mencionar que se realizó con los trabajos preliminares; es decir, visitar al distrito de Shanao, conversar con las autoridades del distrito. Luego de conversar con las autoridades del pueblo, explicando la importancia de la comercialización de los desechos, inclusive tratar de generar energía eléctrica y se planteó la ejecución del proyecto de investigación, aceptó el alcalde y se desarrolló con buenos resultados. A plantear los hallazgos, tuvo que pasar por una etapa de análisis de los datos recabados, en donde empleó el SPSS ver 24, a ello adiciono como estadístico al coeficiente de Pearson. Donde permitió determino el nivel de acercamiento de las variables empleadas. El coeficiente muestra un rango de -1 y 1 , en donde se identifica dependencia directa o indirecta, cuando el valor es 0 indica independencia (Valderrama, 2016).

\section{RESULTADOS Y DISCUSIÓN}

\subsection{RESULTADOS}

Tabla 1: Nivel de desechos domiciliarios por la municipalidad distrital de Shanao, 2019.

\begin{tabular}{|c|c|c|c|}
\hline Escala & Intervalo & $\mathbf{N}^{\circ}$ & $\%$ \\
\hline Pésima & $15-27$ & 23 & $18 \%$ \\
\hline Deficiente & $28-39$ & 27 & $21 \%$ \\
\hline Regular & $40-51$ & 42 & $32 \%$ \\
\hline Buena & $52-63$ & 22 & $17 \%$ \\
\hline Excelente & $64-75$ & 17 & $12 \%$ \\
\hline \multicolumn{2}{|c|}{ Total } & 131 & $100 \%$ \\
\hline
\end{tabular}

Fuente: Cuestionario aplicado a pobladores

\section{Interpretación:}

Se observa el nivel desechos domiciliarios por la municipalidad distrital de Shanao, encontrando, "Regular" de 32\% (42), "Deficiente" con 21\% (27). Asimismo, "Pésimo" con 
$18 \%$ (23), nivel "Bueno" con 17\% (22), y "Excelente" con 12\% (17); donde el nivel "Regular" es el que predomina.

Tabla 2: Nivel de desarrollo local gestionado por la municipalidad distrital de Shanao, 2019.

\begin{tabular}{|c|c|c|c|}
\hline Escala & Intervalo & $\mathbf{N}^{\circ}$ & $\%$ \\
\hline Pésima & $15-27$ & 15 & $11 \%$ \\
\hline Deficiente & $28-39$ & 18 & $14 \%$ \\
\hline Regular & $40-51$ & 35 & $26 \%$ \\
\hline Buena & $52-63$ & 40 & $31 \%$ \\
\hline Excelente & $64-75$ & 23 & $18 \%$ \\
\hline \multicolumn{2}{|c|}{ Total } & 131 & $100 \%$ \\
\hline
\end{tabular}

Fuente: Cuestionario aplicado a pobladores

\section{Interpretación:}

El desarrollo local por la municipalidad distrital de Shanao, alcanzado un nivel "Bueno" de $31 \%$ (40), "Regular" con 26\% (35). Asimismo, "Excelente" con un 18\% (23), un nivel "Deficiente" con un 14\% (18), y el nivel "Pésimo" con un 11\% (15); siendo el nivel "Bueno" que tiene mayor predominancia.

Previamente se formuló la siguiente:

Ho: Manejo de desechos domiciliarios no se relaciona significativamente con el desarrollo local en la Municipalidad Distrital de Shanao, 2019

H1: Manejo de desechos domiciliarios no se relaciona significativamente con el desarrollo local en la Municipalidad Distrital de Shanao, 2019

\section{Nivel de significación:}

Significancia teórica es $\alpha=0,05$; que corresponde a un nivel de confiabilidad del 95\%.

Por tanto: se debe rechazar la Ho cuando la significación observada " $p$ " es menor a $\alpha$. Y no se rechaza la $\mathrm{H}_{1}$ con un " $\mathrm{p}$ " es mayor a $\alpha$. 
Tabla 3: Correlación de desechos domiciliarios y desarrollo local en la municipalidad distrital de Shanao, 2019

\begin{tabular}{ll}
\hline \multicolumn{2}{c}{ Estadísticas de la correlación } \\
\hline Coeficiente de correlación & 0.905 \\
Coeficiente de determinación $\mathrm{R}^{\wedge} 2$ & 0.820 \\
$\mathrm{R}^{\wedge} 2$ ajustado & 0.819 \\
Error típico & 6.420 \\
Observaciones & 131 \\
\hline
\end{tabular}

Fuente: Base de datos trabajado en SPSS ver 24.

Figura 1. Dispersión entre desechos domiciliarios y desarrollo local en la municipalidad distrital de Shanao, 2019

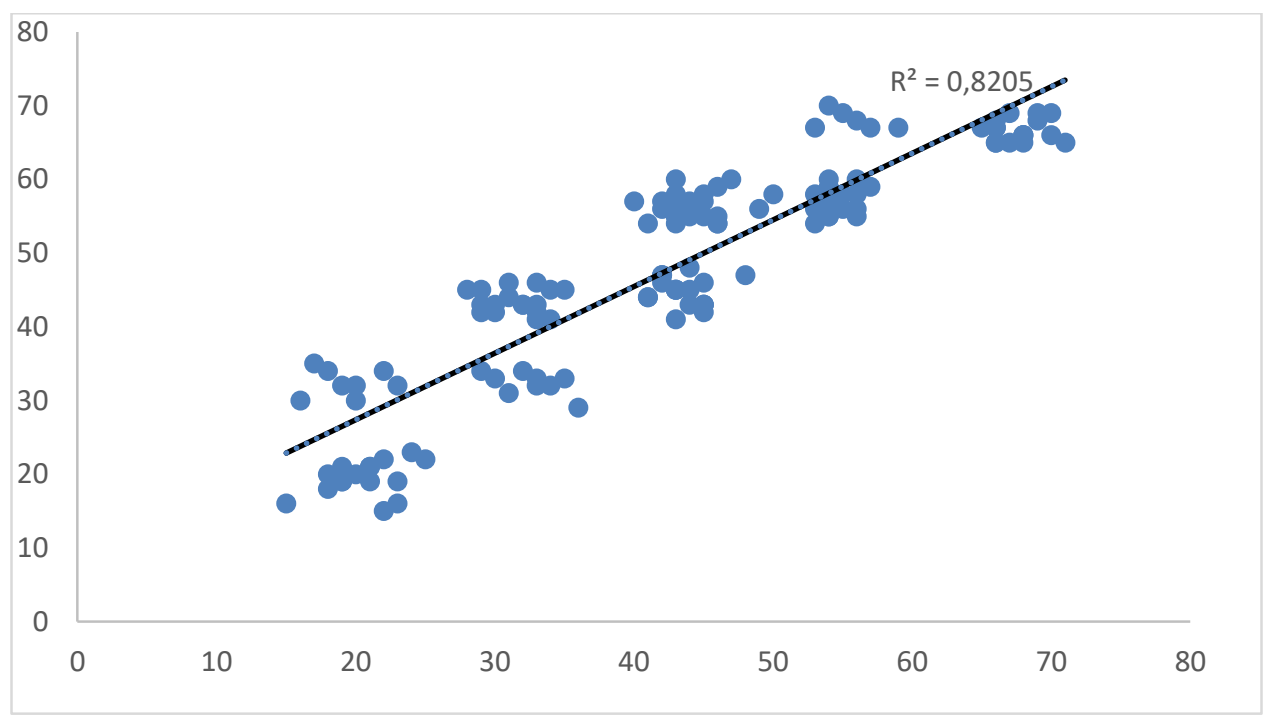

Fuente: Base de datos trabajado en SPSS ver 24.

\section{Interpretación:}

Se encuentra un coeficiente de Pearson de 0.905 , afirmando una relación alta positiva entre el manejo de desechos domiciliarios y desarrollo local en la municipalidad distrital de Shanao. Asimismo, muestra un coeficiente de determinación (0.820) donde 82\% del desarrollo local es influenciado por el manejo de desechos domiciliarios.

\subsection{DISCUSIÓN}

El estudio destaca entre sus hallazgos el nivel desechos domiciliarios gestionados por la municipalidad distrital de Shanao, encontrando un 32\% como "Regular", "Deficiente" con 
21\%. Asimismo, "Pésimo" con 18\%, "Bueno" con 17\%, y "Excelente" con 12\%; destacando el nivel "Regular" por obtener el mayor valor porcentual. Este hallazgo nos permite inferir que los desechos domiciliarios carece del reconocimiento que tiene para las familias, ya que solo muestra un porcentaje mayor en el regular, donde resalta un porcentaje de un $21 \%$, catalogado con nivel deficiente; ante este panorama, hace necesario la intervención del gobierno local, pero para ello debe existir decisión institucional, para poder priorizado y poder asignar los recursos necesarios para poder intervenir, donde además la intervención tiene que ser colaborativa y con una alta participación comunitaria, ya que pueda permitir desarrollar trabajos organizados y convergentes. Dicho resultado al ser comparado con Lozano (2019) donde concluyó que el sistema gestión de desechos domiciliarios alcanzo un 14\%, la gestión integral, con 79\%; en donde se identifica procesos con una valoración regular. Este resultado, difiere significativamente con el hallazgo del estudio, ya sus resultados son menores al resultado de Lozano.

Asimismo, el autor Alfaro (2008), hace mención de la responsabilidad del gobierno local del manejo de los desechos generados en la jurisdicción mediante su área correspondiente del gobierno local (p. 24). De acuerdo a lo mencionado, todo gobierno municipal tiene la responsabilidad de gestionar sus residuos sólidos, los mismos que fueron generados por la comunidad, sectores, instituciones y otros que se encuentren en la circunscripción geográfica. Por tanto, los desechos de residuos domiciliarios es función y responsabilidad del consejo y alcalde de la municipalidad para poder atender esta problemática, que cada día es común en los distritos y provincias, y que aún es un tema que debe ser abordado de manera responsable. Asimismo, el estudio muestra el desarrollo local, encontrando un nivel "Bueno" de 31\%, "Regular" con 26\%, "Excelente" con 18\%, "Deficiente" con 14\%, y "Pésimo" con un 11\%; siendo el nivel "Bueno" que tiene mayor predominancia. De acuerdo a este hallazgo, se infiere que esta variable es mejor valorada por los pobladores, básicamente por el asfalto de sus calles y el mejoramiento de las instituciones educativas; sin embargo, hay un buen porcentaje de pobladores que refieren niveles deficientes, el mismo que debe ser considerado como una petición de la ciudadanía y resuelto posteriormente, mediante acciones concretas que verdaderamente propicie efectos positivos en la comunidad. 
Es necesario precisar que el desarrollo local, es preciso considerar el espacio nacional, para considerar un desarrollo local, debe contener seis ejes estratégicos para realizar una mirada multidimensional, en donde la participación de los gobiernos locales es fundamental, debiendo considerarse infraestructura y servicios educativos y sanitarios; donde puedan complementarse para un buen desarrollo (CEPLAN, 2011).

Por tanto, el desarrollo local este articulado con un crecimiento país, para ello es necesario que el gobierno local pueda ser convocante a los sectores públicos instalados en el distrito para generar un plan con mirada prospectiva, donde puedan generarse objetivos comunes, y con ello poder planificar y desarrollar actividades, haciendo uso eficiente de los recursos públicos. Asimismo, debe existir la participación y el involucramiento de la parte privada, ya que es actor de gran importancia para generar un desarrollo armónico.

Finalmente, el principal hallazgo donde presenta una relación alta positiva entre el manejo de desechos domiciliarios y desarrollo local, sustentado con un coeficiente de Pearson de 0.905; un coeficiente de determinación (0.820) donde 82\% del desarrollo local es influenciado por el manejo de desechos domiciliarios. Al contrastar con Mori (2018) donde concluyo que existe una correlación significativa alta positiva entre la recaudación tributaria y desarrollo local.

En base en sus dos hallazgos se infiere que el desarrollo local, es una variable de gran relevancia para la ciudadanía, ya que es la medición de sus autoridades locales, donde surge mediante la gestión de sus autoridades y sus repercusiones que tienes, para ello es necesario propiciar proyectos vinculados con las necesidades de la comunidad y sus habitantes, para ello es necesario contar con una población que participe activamente como parte de su deber ciudadano, en ese sentido, es el gobierno local el principal convocante para articular las necesidades con posibles proyectos que han sido planificados dentro sus documentos de gestión (PEI, POI).

Al comparar con Bernal (2014), donde menciona que para un crecimiento de un país se debe considerar una variedad de deficiencias, como es el caso de la inestabilidad económica, ya que esta repercute a la región, y con consecuencias a los distritos como menor nivel de territorio nacional. En ese sentido, el desarrollo también está vinculado con variables externas que en circunstancias los gobiernos locales no alcanzar a poder control, ya que su acción 
gubernamental le impide realizar acciones. Por tanto, el desarrollo tiene que producir en condiciones adecuadas para poder ser generadora de impacto socio económico en las familias, como unidad básica de la sociedad.

\section{CONCLUSIONES}

El manejo de desechos domiciliarios y desarrollo local tiene relación alta positiva, sustentado por coeficiente de Pearson de 0.905, y con coeficiente de determinación (0.820), evidencia un $82 \%$ del desarrollo local viene influenciado por el manejo de desechos domiciliarios de la municipalidad distrital de Shanao.

Los desechos domiciliarios gestionados por la municipalidad distrital de Shanao, tiene nivel "Regular" de 32\%, "Deficiente" con 21\%. Asimismo, un nivel "Pésimo" con 18\%, un nivel "Bueno" con un 17\%, y el "Excelente" con 12\%; siendo el nivel "Regular" una mayor predominancia.

El desarrollo local por la municipalidad distrital de Shanao, muestra nivel "Bueno" de 31\%, "Regular" con 26\%, "Excelente" con 18\%, "Deficiente" con 14\%, y nivel "Pésimo" con $11 \%$; siendo el nivel "Bueno" muestra mayor predominancia.

Al alcalde y al consejo municipal del Distrito de Shanao, promover e institucionalizar un plan integral de manejo de desecho domiciliario y el mismo que se vincule con el desarrollo urbano del distrito mediante la promoción del turismo local.

\section{REFERENCIAS}

Alfaro, D. (2008). Huella Ecológica de las Ciudades del Perú. Construyendo Ciudades para la Vida:Aporte a la Construcción Sostenible en el Perú. Lima: Foro ciudades para la Vida.

Cepal - Ilpes. (2003). Descentralización en América Latina: cómo hacer viable el desarrollo local. Santiago de Chile: Ilpes - Cepal.

Cepal. (2004). División de Recursos Naturales e Infraestructura. Santiago de Chile: Nacionaes Unidas.

CEPLAN. (2011). Plan Bicentenario: El Perú hacia el 2021. Lima: Biblioteca Nacional del Perú.

D.S. N 057-2004-PCM. (2004). Reglamento de la Ley General de Residuos Sólidos. Lima. 
Dirección General de Políticas. (2009). Normativa ambiental peruana. Lima: Dirección General de Políticas.

Entrelíneas. (2013). Tributación Municipal. Asesoría Empresarial, 57.

George, D., \& Mallery, P. (2003). SPSS for Windows step by step: A simple guide and reference. 11.0 update (4th ed.). Boston: Allyn \& Bacon.

Hernández, R. (2016). Metodología de la Investigación. Lima: Mc Graw Hill.

IFC, \& ACDI. (2012). Inversión municipal y rendición social de cuentas. Lima: GMC Gráfica S.A.C.

INEI. (2007). Censos nacionales 2007. XI de población y VI de viviendda. Lima: INEI.

Karak, \&. e. (2012). a Gestión Integral de los Residuos Sólidos en México. México.

Ley N²7314. (2000). Ley General de Residuos Sólidos. Ley General de Residuos Sólidos. Lima, Perú.

Lozano, A. (2019). Sistema de gestión basado en métodos de residuos sólidos para mejorar el manejo de desechos domiciliarios en Cuñmbuqui-Lamas-2018. Tarapoto: Universidad César Vallejo.

Marin, R., Barreix, A., \& Machado, R. (2015). Recaudar para crecer: bases para la reforma tributaria en Centroamérica (1era ed.). México: IDB.

Marquez , J., \& Rovira, A. (2002). El desarrollo local.

Márquez, J. (2011). Metodología del desarrollo local. Estrategias e instrumentos. Huelva: Universidad Internacional de Andalucía.

MEF. (29 de Abril de 2016). Sistema nacional de inversión pública. Lima, Lima, LIma .

MINAM. (2016). Plan Nacional de Gestión Integral de Residuos Sólidos. Lima: MINAM.

Montes, C. (2009). Régimen jurídico y ambiental de los residuos sólidos. Colombia: Universidad Externado de Colombia.

Mori, P. (2018). Relación de la recaudación tributaria con el desarrollo local gestionado por la municipalidad distrital de Barranquita, 2018. Tarapoto: Universidad César Vallejo. 
Pinto, M. (2009). Régimen jurídico y ambiental de los residuos sólidos. Colombia: Universidad Externadode Colombia.

PROARCA. (2003). Guía para la gestión del manejo de los residuos sólidos municipales: Enfoque Centro América. Guatemala: Programa Regional Ambiental para Centroamérica.

Programa de las Naciones Unidas para el Medio Ambiente. (2011). Perpectiva del medio ambiente mundial. Panama: Programa de las Naciones Unidas para el Medio Ambiente.

Semarnat. (2003). Secretaria de Medio Ambiente y Recursos Naturales. México: Semarnat.

USAID. (2011). Manual para la mejora de la recaudación del impuesto predial en las municipalidades. Lima: Nuevo Studio S.A.C.

Valderrama, S. (2016). Pasos para elaborar proyectos de investigación científica (6 ta ed.). Lima: San Marcos. 